\title{
Desempenho de cultivares de feijoeiro irrigado no ecótono Cerrado-Amazônia
}

\author{
Matheus Bortolanza SOARES ${ }^{1}$, Joaquim Pedro de LIMA $^{2}$, Onã da Silva FREDDI ${ }^{2 *}$, \\ Tauan Rimoldi TAVANTI ${ }^{3}$, Renan Francisco Rimoldi TAVANTI ${ }^{4}$, Flavio Jesus WRUCK ${ }^{5}$
}

\author{
${ }^{1}$ Departamento de Ciência do Solo, Escola Superior de Agricultura "Luiz de Queiroz”, Universidade de São Paulo, Piracicaba, SP, Brasil. \\ ${ }^{2}$ Instituto de Ciências Agrárias e Ambientais, Universidade Federal de Mato Grosso, Sinop, MT, Brasil. \\ ${ }^{3}$ Departamento de Fitotecnia, Tecnologia de Alimentos e Sócio Economia, Universidade Estadual Paulista, Ilha Solteira, SP, Brasil. \\ ${ }^{4}$ Departamento de Fitossanidade, Engenharia Rural e Solos, Universidade Estadual Paulista, Ilha Solteira, SP, Brasil. \\ ${ }^{5}$ Embrapa Agrossilvipastoril, Sinop, MT, Brasil. \\ *E-mail: onafreddi@gmail.com
}

Recebido em abril/2018; Aceito em novembro/2018.

\begin{abstract}
RESUMO: O uso da irrigação por pivô central favorece o cultivo do feijoeiro de terceira safra, contudo, limitações à produtividade são observadas quando cultivados em sistema de semeadura direta, sendo a compactação superficial, incidência de nematoides e variabilidade espacial vertical e horizontal dos nutrientes do solo os principais fatores limitantes deste sistema. O objetivo do estudo foi avaliar o desempenho de cultivares de feijoeiro irrigado em terceira safra, cultivados em sistema de semeadura direta e sua correlação com a fertilidade do solo e incidência de nematoides na região de ecótono Cerrado-Amazônia. O experimento foi desenvolvido no município de Sorriso-MT em um Latossolo Vermelho-Amarelo Distrófico. Os tratamentos foram constituídos por quatro cultivares de feijão, sendo eles: BRS Estilo, BRS Notável, BRS Ametista e BRS Pérola, em uma área total de $9900 \mathrm{~m}^{2}$, onde se determinou através de 83 pontos amostrais georreferenciados os atributos de planta e atributos químicos do solo. Os procedimentos estatísticos utilizados foram: análise descritiva dos dados, análise geoestatística e comparação de médias pelo método "bootstrap". A maioria dos atributos apresentaram moderada à alta dependência espacial, com elevada variabilidade espacial. $\mathrm{O}$ aumento dos teores de potássio gerou incrementos na produtividade, enquanto que o aumento da incidência de nematoides, acidez potencial e saturação de alumínio no solo resultaram em redução. A maior produtividade observada foi da cultivar BRS Pérola, indicando boa aptidão para cultivo nesta região.
\end{abstract}

Palavras-chave: Phaseolus vulgaris L., fertilidade do solo, nematóide, geoestatística.

\section{Performance of irrigated bean cultivars in the Cerrado-Amazonian ecotone}

\begin{abstract}
The use of central pivot irrigation favors third-crop bean cultivation, however, productivity limitations are observed when cultivated in a no-tillage system, with surface compaction, nematode incidence and vertical and horizontal spatial variability of soil nutrients, main limiting factors of this system. The objective of this study was evaluate the performance of third-crop irrigated bean cultivars grown under no-tillage system and the correlation with soil fertility and nematode incidence in the Cerrado-Amazonian ecotone region. The experiment was developed in the Sorriso-MT county in a Dystrophic Red Yellow Latosol. The treatments consisted of four bean cultivars: BRS Estilo, BRS Notável, BRS Ametista and BRS Pérola, in a total area of 9900 $\mathrm{m}^{2}$, where soil chemical and plant attributes were determined through 83 georeferenced sampling points. The statistical procedures used were: descriptive analysis of the data, geostatistical analysis and comparison of means by the "bootstrap" method. Most of the attributes presented moderate to high spatial dependence, with high spatial variability. The increase of potassium contents generated increases in productivity, while the increase in the incidence of nematodes, potential acidity and aluminum saturation in the soil resulted in a reduction. The highest productivity observed was of the cultivar BRS Pérola, indicating good aptitude for cultivation in this region.
\end{abstract}

Keywords: Phaseolus vulgaris L., soil fertility, nematode, geoestatistics.

\section{INTRODUÇÃO}

O feijão possui especial importância para a agricultura brasileira frente sua relevância na dieta da população, sendo o país o terceiro maior produtor mundial (CONAB, 2017). Isso é possível graças ao cultivo de três safras durante ano agrícola, principalmente em terceira safra, na qual o uso da irrigação é imprescindível (GOMES et al., 2013), por se tratar de um período de seca, sendo esta safra do feijoeiro mais tecnificada, com maiores produtividades e melhores preços de mercado.

Produtores da região Centro-Oeste aumentaram seu interesse pela cultura, mais especificamente no estado de Mato
Grosso, que possui área irrigada por pivô central estimada de 80.107 ha (ANA, 2014), alcançando produtividade média de $2.103 \mathrm{~kg} \mathrm{ha}^{-1}$ (CONAB, 2017). Contudo, ainda se observa alguns desafios para se obter produtividades superiores a 3000 $\mathrm{kg} \mathrm{ha}^{-1}$ (MONTANARI et al., 2010). Os principais fatores limitantes a produtividade do feijoeiro são: a compactação superficial do solo, devido ao sistema de semeadura direta, e ausência de rotação de culturas, resultando em elevados valores de resistência do solo à penetração e baixos valores de infiltração de água (HOU et al., 2012; SOUZA et al., 2014). A incidência e aumento populacional de nematoides em áreas 
irrigadas também é outro fator (CONAB, 2016), ocorre principalmente pelo uso contínuo de lavouras com plantas hospedeiras; e gradientes horizontais e verticais da fertilidade do solo, justificados pelas práticas de adubações a lanço, resultando em elevada variabilidade dos nutrientes neste sistema (SILVA; CHAVES, 2001).

Alguns estudos foram realizados visando esclarecer a relação entre a produtividade do feijão e a variabilidade dos atributos do solo (AMADO et al., 2009; DALCHIAVON et al., 2011), tendo consenso quanto ao fato de que a produtividade está fortemente relacionada com vários componentes da fertilidade do solo, como o $\mathrm{pH}$, fósforo $(\mathrm{P})$, potássio $(\mathrm{K})$, cálcio $(\mathrm{Ca})$,magnésio $(\mathrm{Mg})$, capacidade de troca de cátions a pH 7,00 (T), saturação por bases (V\%) e acidez potencial (H+Al). Da mesma forma, Machado (2011) explica em seus estudos que perdas na produtividade entre $10 \%$ a $50 \%$ para o feijoeiro ocorrem principalmente quando atacado por nematoides.

Cultivares adaptadas ao Cerrado brasileiro estão sendo testadas buscando aliar altas produtividades aos desafios já mencionados. O sistema de produção da região Centro-Oeste do Brasil tem gerado novos questionamentos para a otimização da produção agrícola. Neste sentido, o objetivo do estudo foi avaliar o desempenho de cultivares de feijoeiro irrigado em terceira safra, cultivados em sistema de semeadura direta e sua correlação com a fertilidade do solo e incidência de nematoides na região de ecótono Cerrado-Amazônia.

\section{MATERIAL E MÉTODOS}

O experimento foi desenvolvido na Fazenda Vitória, situada no município de Sorriso, norte do Estado de Mato Grosso, nas coordenadas $12^{\circ} 33^{\prime} 31^{\prime \prime} \mathrm{S}$ e $55^{\circ} 42^{\prime} 51^{\prime \prime} \mathrm{O}$, com altitude média de 386 metros. O clima da região é do tipo tropical úmido (Aw, segundo classificação de Köppen), com precipitação anual em torno de $2000 \mathrm{~mm}$.

O solo foi classificado segundo o Sistema Brasileiro de Classificação do Solo (Embrapa 2018), como Latossolo Vermelho-Amarelo Distrófico de textura franco argilo arenosa, com teores de $705 \mathrm{~g} \mathrm{~kg}^{-1}$ de areia, $74 \mathrm{~g} \mathrm{~kg}^{-1}$ de silte, $221 \mathrm{~g} \mathrm{~kg}^{-1}$ de argila e $24,3 \mathrm{~g} \mathrm{~kg}^{-1}$ de matéria orgânica na camada de 0,00-0,20 m. A área vem sendo utilizada em sistema de semeadura direta há 5 anos com as culturas da soja na primeira safra, milho segunda safra e feijão na terceira safra. Os tratamentos foram constituídos por quatro cultivares de feijão do grupo carioca, sendo elas: BRS Estilo, BRS Notável, BRS Ametista e BRS Pérola. As cultivares utilizadas foram semeadas mecanicamente com espaçamento de $0,45 \mathrm{~m}$ entre linhas, com adubação de $600 \mathrm{~kg} \mathrm{ha}^{-1}$ do formulado $05-$ 25-25, aplicados a lanço.

O controle de plantas daninhas e prevenção das principais pragas e doenças do feijoeiro foram realizados por meio de pulverizações com produtos recomendados para a cultura, sendo eles os herbicidas Imazetapir e Flex; fungicidas Fox, Propiconazole, Impact e Aureo, e inseticidas Nomolt, Acefato, Connect e Belt, ambos nas doses recomendadas para a cultura. A irrigação foi realizada por pivô central de acordo com as exigências da cultura, sendo aplicado no total $127 \mathrm{~mm}$ de água durante o experimento.

Para amostragem dos atributos de solo e planta, instalouse uma malha retangular, constituída de quatro linhas e 12 colunas, contendo 48 pontos amostrais, espaçados de 20 em 20 m entre linhas e 15 em 15 m entre colunas, totalizando uma área de $9900 \mathrm{~m}^{2}$ (Figura 1).

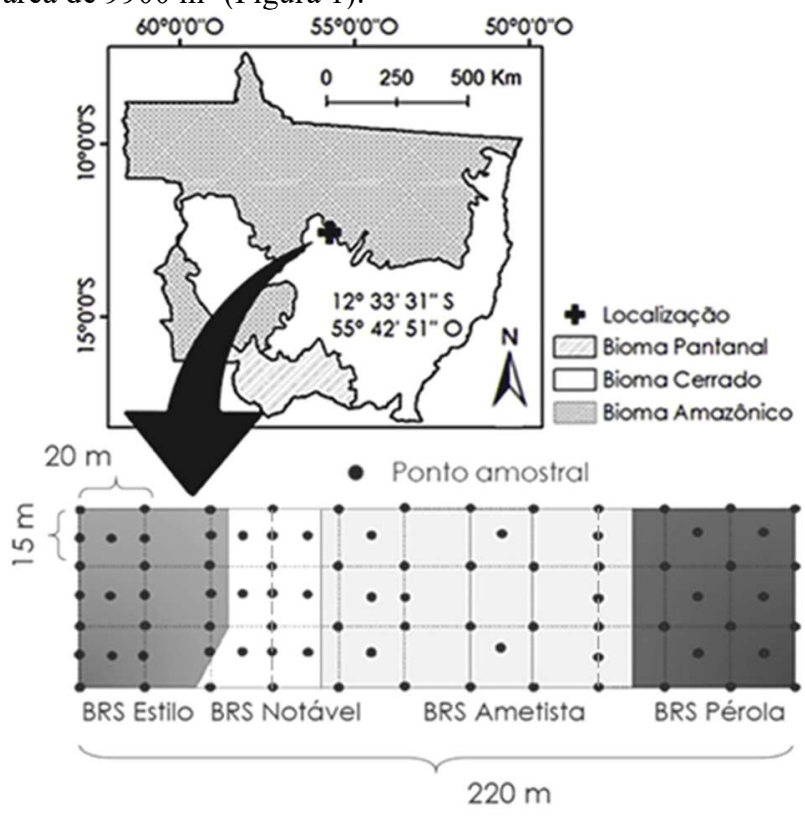

Figura 1. Local do estudo e ilustração da malha amostral contento 83 pontos georreferenciados e os respectivos tratamentos.

Figure 1. Site of the study and illustration of the sampling mesh content 83 georeferenced points and the respective treatments.

Além disso, foram alocados dentro da malha mais 35 pontos de refinamento, visando detalhar o estudo da dependência espacial dos dados para espaçamento entre pontos menores do que $20 \mathrm{~m}$. Somando-se os pontos da malha e os de refinamento o total foi de 83 pontos amostrais. Os tratamentos BRS Estilo, BRS Notável, BRS Ametista e BRS Pérola ocuparam área de 2175, 1825, 3600, $2300 \mathrm{~m}^{2}$, respectivamente.

Durante o período de colheita e nas entrelinhas de semeadura do feijão, coletaram-se amostras deformadas do solo com auxílio de um trado holandês, na camada de 0-0,20 $\mathrm{m}$, para determinação de $\mathrm{pH}$ em $\mathrm{H}_{2} \mathrm{O}$, teores de fósforo disponível $(\mathrm{P})$, potássio trocável $(\mathrm{K})$, cálcio trocável $(\mathrm{Ca})$, magnésio trocável $(\mathrm{Mg})$, alumínio trocável $(\mathrm{Al})$, acidez potencial $(\mathrm{H}+\mathrm{Al})$ e matéria orgânica $(\mathrm{MO})$. Sendo $\mathrm{P}$ e $\mathrm{MO}$ determinados por colorimetria, $\mathrm{Ca}$ e $\mathrm{Mg}$ determinados por espectrofotometria de absorção atômica e $\mathrm{H}+\mathrm{Al}$ e $\mathrm{Al}$ determinados por titulação alcalimétrica, através dos extratores cloreto de potássio e acetato de cálcio, respectivamente, e $\mathrm{pH}$ determinado em água conforme a metodologia descrita pela Embrapa (2011). Os atributos químicos de solo calculados foram: soma de bases (SB), saturação por bases $(\mathrm{V} \%)$, capacidade efetiva de troca de cátions $(\mathrm{t})$, capacidade de troca de cátions à $\mathrm{pH} 7(\mathrm{~T}) \mathrm{e}$ saturação por alumínio $(\mathrm{m} \%)$, conforme metodologia descrita pela Embrapa (2011).

Para a determinação da produtividade de grãos $(\mathrm{PG})$ e número de plantas por parcela $(\mathrm{NP})$, coletaram-se em cada ponto amostral duas linhas de $3 \mathrm{~m}$, totalizando uma área útil de $2,7 \mathrm{~m}^{2}$. A produtividade de grãos foi corrigida para umidade de $13 \%$ e representada em $\mathrm{kg} \mathrm{ha}^{-1}$. Em 10 plantas da área útil determinou-se a altura de planta (ALT), altura de inserção da primeira vagem (INS), número de vagens (NVG), massa de cem grãos (M100), e nota de incidência de galhas nas raízes (NT). 
A coleta das raízes para avaliação da incidência de nematoides ocorreu por ocasião da colheita do feijão. As raízes foram coletadas manualmente e armazenadas em sacos plásticos. Para avaliar o grau de incidência de galhas, adotouse a escala de Taylor; Sasser (1978), cuja escala de notas varia de 0 a 5 de acordo com o número de galhas visíveis, sendo nota $0=0$ galhas visíveis, $1=1$ ou 2 galhas visíveis, $2=3$ a 10 galhas visíveis, $3=11$ a 30 galhas visíveis, $4=31$ a 100 galhas visíveis e 5 = mais que 100 galhas visíveis.

Os dados foram submetidos a análise descritiva para estudo explanatório de variáveis e a hipótese de normalidade foi verificada pelo teste de Shapiro-Wilk $(p<0.05)$. Em seguida, procedeu-se a análise geoestatística dos dados pela modelagem dos semivariogramas teóricos.

A classificação do avaliador de dependência espacial (ADE) foi feita com base na razão entre o efeito pepita e o patamar $(\mathrm{C} / \mathrm{C}+\mathrm{Co})$, segundo o qual $\mathrm{ADE} \leq 20 \%$ indica variável com muito baixa dependência espacial; $20 \%<\mathrm{ADE}$ $\leq 40 \%$ indica variável com baixa dependência espacial; $40 \%<$ $\mathrm{ADE} \leq 60 \%$ variável com média dependência espacial; $60 \%<$ $\mathrm{ADE} \leq 80 \%$ variável com alta dependência espacial; e ADE $>$ $80 \%$ variável com dependência espacial muita alta (DALCHIAVON et al., 2011). O critério final de decisão do modelo de semivariograma experimental e do número de vizinhos usados na predição de valores e o melhor ajuste obtido pela validação cruzada. Uma vez ajustados os semivariogramas, realizou-se a krigagem ordinária dos dados e a composição de mapas de contorno com intervalos equidistantes.

As médias dos tratamentos foram comparadas pela técnica de "bootstrap", com 1.000 reamostragens aleatórias com reposição, conforme o método descrito por Christie (2004). A partir do universo de 1.000 valores, também foi possível estabelecer os limites superior e inferior do intervalo de confiança da média, com $95 \%$ de probabilidade, tendo sido esse procedimento útil para a posterior comparação das médias entre si (MELLO et al., 2015). Assim, médias com valores comuns dentro de seus intervalos de confiança, cujas barras de erro se encontram, não diferem entre si, ao passo que as ausências de valores comuns indicam diferença significativa (a $5 \%$ de probabilidade) entre elas.

\section{RESULTADOS}

Conforme o coeficiente de variação classificado por Pimentel Gomes; Garcia (2002), observou-se variabilidade média a muito alta para os atributos do feijão, com destaque para nota de incidência de galhas nas raízes, com coeficiente de variação de $64,02 \%$, sinalizando que entre as cultivares houve grande variação nos resultados (Tabela 1)

A PG obtida neste estudo foi de $1810 \mathrm{~kg} \mathrm{ha}^{-1}$, com elevada amplitude de valores mínimo e máximo, sendo observado locais com produtividades de $700 \mathrm{~kg} \mathrm{ha}^{-1}$ e outros com até $3040 \mathrm{~kg} \mathrm{ha}^{-1}$. Isso demonstra que possivelmente as cultivares de feijoeiro apresentaram diferentes adaptações as condições do solo e incidência de nematoides.

Tabela 1. Análise descritiva dos atributos de planta e dos atributos químicos do Latossolo Vermelho-Amarelo Distrófico cultivado com feijão em sistema de semeadura direta.

Table 1. Descriptive analysis of plant attributes and chemical attributes of the Dystrophic Red Yellow Latosol cultivated with beans in notillage system.

\begin{tabular}{|c|c|c|c|c|c|c|c|c|c|c|}
\hline \multirow{3}{*}{ Atributo $^{(a)}$} & \multicolumn{10}{|c|}{ Medidas Estatísticas Descritivas } \\
\hline & \multicolumn{5}{|c|}{ Valor } & \multicolumn{3}{|c|}{ Coeficiente $^{(\mathrm{C})}$} & \multicolumn{2}{|c|}{ Prob. $^{(d)}$} \\
\hline & Média & Mediana & Mínimo & Máximo & $\mathrm{DP}^{(\mathrm{b})}$ & $\mathrm{CV}(\%)$ & Curtose & Ass. & $\mathrm{Pr}<\mathrm{W}$ & $\mathrm{DF}$ \\
\hline & \multicolumn{10}{|c|}{ Atributos da planta } \\
\hline NP & 36,90 & 35,00 & 24,00 & 54,00 & 8,70 & 23,81 & $-1,19$ & 0,40 & 0,00 & TN \\
\hline NT & 2,90 & 3,50 & 0,00 & 5,00 & 1,80 & 64,02 & $-1,40$ & $-0,41$ & 0,00 & $\mathrm{TN}$ \\
\hline ALT & 57,50 & 56,80 & 35,10 & 87,60 & 10,50 & 18,29 & 0,49 & 0,51 & 0,13 & NO \\
\hline INS & 20,50 & 20,30 & 9,90 & 34,10 & 4,50 & 22,30 & 0,68 & 0,64 & 0,02 & $\mathrm{TN}$ \\
\hline NVG & 12,60 & 12,80 & 6,80 & 20,80 & 2,91 & 23,03 & $-0,20$ & 0,27 & 0,41 & NO \\
\hline M100 & 25,39 & 25,00 & 19,00 & 32,00 & 2,55 & 10,06 & 0,01 & $-0,03$ & 0,07 & NO \\
\hline \multirow[t]{2}{*}{ PG } & 1810 & 1780 & 700 & 3040 & 575 & 31,71 & $-0,89$ & 0,01 & 0,10 & NO \\
\hline & \multicolumn{10}{|c|}{ Atributos químicos do solo } \\
\hline$P$ & 63,49 & 66,15 & 5,43 & 91,90 & 18,20 & 28,66 & $-0,07$ & $-0,66$ & 0,00 & $\mathrm{TN}$ \\
\hline K & 39,71 & 38,94 & 20,11 & 62,61 & 9,64 & 24,27 & $-0,37$ & 0,36 & 0,26 & NO \\
\hline $\mathrm{Ca}$ & 1,38 & 1,41 & 0,42 & 2,48 & 0,39 & 28,41 & $-0,15$ & 0,04 & 0,97 & NO \\
\hline $\mathrm{Mg}$ & 0,82 & 0,81 & 0,65 & 1,01 & 0,08 & 10,18 & $-0,43$ & 0,34 & 0,07 & NO \\
\hline SB & 2,31 & 2,35 & 1,19 & 3,74 & 0,48 & 21,07 & $-0,07$ & 0,15 & 0,91 & NO \\
\hline $\mathrm{Al}$ & 0,21 & 0,21 & 0,04 & 0,39 & 0,07 & 35,67 & $-0,01$ & 0,30 & 0,27 & NO \\
\hline $\mathrm{H}+\mathrm{Al}$ & 5,40 & 5,28 & 3,46 & 7,70 & 0,92 & 17,10 & $-0,48$ & 0,28 & 0,45 & NO \\
\hline $\mathrm{t}$ & 2,52 & 2,54 & 1,37 & 3,78 & 0,43 & 17,39 & 0,02 & 0,06 & 0,97 & NO \\
\hline $\mathrm{T}$ & 7,72 & 7,58 & 4,66 & 10,58 & 1,10 & 14,32 & 0,01 & $-0,07$ & 0,52 & $\mathrm{NO}$ \\
\hline V\% & 30,04 & 29,63 & 18,54 & 42,13 & 5,19 & 17,29 & $-0,13$ & 0,18 & 0,58 & NO \\
\hline $\mathrm{m} \%$ & 8,74 & 8,31 & 1,05 & 18,18 & 3,96 & 45,31 & $-0,55$ & 0,36 & 0,12 & NO \\
\hline $\mathrm{pH}$ & 5,80 & 5,79 & 5,15 & 6,42 & 0,35 & 6,15 & $-1,15$ & 0,02 & 0,00 & $\mathrm{TN}$ \\
\hline MO & 24,30 & 24,10 & 0,89 & 38,80 & 0,52 & 21,44 & 0,65 & 0,14 & 0,70 & NO \\
\hline
\end{tabular}

(a) NP: número de planta; NT: nota de incidência de galhas nas raízes; ALT: altura de planta (cm); INS: altura de inserção da primeira vagem (cm); NVG: número de vagem por planta; M100: massa de cem grãos (g); PG: produtividade de grãos de feijão ( $\left.\mathrm{kg} \mathrm{ha}^{-1}\right)$; P: Fósforo do solo (mg dm $\left.{ }^{-3}\right)$; $\mathrm{K}^{-3}$ Potássio do solo

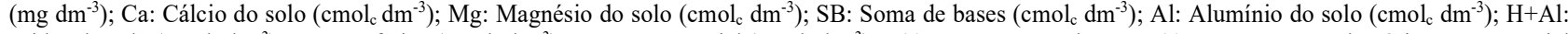
acidez do solo $\left(\mathrm{cmol}_{\mathrm{c}} \mathrm{dm}^{-3}\right)$; t: CTC efetiva $\left(\mathrm{cmol}_{\mathrm{c}} \mathrm{dm}^{-3}\right)$; T: CTC potencial $\left(\mathrm{cmol}_{\mathrm{c}} \mathrm{dm}^{-3}\right)$; V\%: Saturação por bases; $\mathrm{m} \%$ : Saturação por alumínio; pH: Potencial hidrogeniônico em água; MO: Matéria orgânica do solo ( $\left.\mathrm{g} \mathrm{kg}^{-1}\right)$, respectivamente. (b) DP: Desvio Padrão. (c) CV: Coeficiente de variação; Ass.: Assimetria. (d) Prob: Probabilidade referente ao teste de normalidade de Shapiro Wilk; DF: distribuição de frequências, sendo NO do tipo normal e TN tendendo ao normal. 
Os atributos químicos do solo foram classificados em seus teores de acordo com critérios estabelecidos por Sousa; Lobato (2004) para condições do Cerrado brasileiro. O teor médio de $\mathrm{K}$ foi de $39,71 \mathrm{mg} \mathrm{dm}^{-3}$, considerado inadequado para a cultura do feijoeiro. O teor de $\mathrm{P}$ apresentou elevada disponibilidade com valor médio de $63,49 \mathrm{mg} \mathrm{dm}^{-3}$, considerando o teor de argila de $221 \mathrm{~g} \mathrm{~kg}^{-1}$. Esse teor de $\mathrm{P}$ no solo pode ser justificado pelas adubações realizadas ao longo dos anos na fazenda, na qual apresenta solo de fertilidade construída. Os teores médios de $\mathrm{Ca}$ e $\mathrm{Mg}$ foram de 1,38 e 0,82 $\mathrm{cmol}_{\mathrm{c}} \mathrm{dm}^{-3}$, respectivamente, representando uma média disponibilidade no complexo de troca de cátions. $\mathrm{O} \mathrm{Mg} \mathrm{e} \mathrm{o} \mathrm{pH}$ apresentaram a menor variabilidade na área, com coeficientes de variação inferiores a $11 \%$.

O solo apresentou teores de Al trocável de até $0,39 \mathrm{cmol}_{\mathrm{c}}$ $\mathrm{dm}^{-3}$, com saturação de $8,74 \%$ na $\mathrm{t}$, sendo tal condição indesejável para cultura do feijoeiro. $\mathrm{O} \mathrm{Al}$ presente no solo pode ser justificado pelos valores de $\mathrm{pH}$ inferiores a 5,5, justificando os baixos valores de $\mathrm{V} \%$ encontrados na área, indicando também a necessidade de calagem. Devido a isto observa-se um valor médio para acidez potencial de $5,40 \mathrm{cmol}_{\mathrm{c}} \mathrm{dm}^{-3}$.

O teste de Shapiro-Wilk confirmou a distribuição do tipo normal para a maioria dos atributos da planta e do solo, com exceção do NP, NT, INS, P e pH. Embora o teste tenha sido significativo para estas variáveis, indicando desvios da normalidade, considerou-se que estes dados apresentaram distribuição tendendo a normalidade devido aos baixos coeficientes de curtose e assimetria, e a proximidade entre valores médios e a mediana.

Lembrando que, a distribuição de frequência do tipo normal não é necessária para a análise geoestatística, porém é importante que os dados não apresentem caudas longas e/ou dados discrepantes, definidos como "outliers".

As variáveis NT, ALT, INS, NVG, M100, PG, P, K, Mg, $\mathrm{Al}, \mathrm{H}+\mathrm{Al}, \mathrm{T}, \mathrm{m} \%$ e $\mathrm{pH}$ apresentaram de moderada a alta dependência espacial (Tabela 2). Contudo, não houve dependência espacial para a NP, Ca, SB, t, V\% e MO.

Tabela 2. Parâmetros dos semivariogramas simples e cruzados ajustados para os atributos de planta e atributos químicos de um Latossolo Vermelho-Amarelo Distrófico cultivado com feijão sob sistema de semeadura direta.

Table 2. Parameters of the simple and crossed semivariograms adjusted for the attributes of plant and chemical attributes of a Dystrophic Red Yellow Latossol cultivated with beans under no-tillage system.

\begin{tabular}{|c|c|c|c|c|c|c|c|c|c|c|c|}
\hline \multirow{3}{*}{ Atributos } & \multicolumn{11}{|c|}{ Parâmetros do ajuste } \\
\hline & \multirow{2}{*}{ modelo } & \multirow{2}{*}{$\mathrm{C} 0$} & \multirow{2}{*}{$\mathrm{C} 0+\mathrm{C}$} & \multirow{2}{*}{$\mathrm{A} 0(\mathrm{~m})$} & \multirow{2}{*}{$\mathrm{R}^{2}$} & \multirow{2}{*}{$\mathrm{SQR}$} & \multicolumn{2}{|c|}{$\mathrm{ADE}$} & \multicolumn{3}{|c|}{ Validação cruzada } \\
\hline & & & & & & & $\%$ & Classe & $\mathrm{a}$ & $\mathrm{b}$ & $\mathrm{R}$ \\
\hline NP & epp & 77,52 & 77,52 & - & - & - & - & - & - & - & - \\
\hline NT & gau & 1,49 & 3,42 & 42,95 & 0,36 & 3,60 & 56,50 & MO & 0,09 & 0,96 & 0,60 \\
\hline ALT & $\exp$ & 62,80 & 127,80 & 113,40 & 0,78 & 597,0 & 50,90 & MO & 5,89 & 0,90 & 0,41 \\
\hline INS & $\exp$ & 2,66 & 16,43 & 28,80 & 0,26 & 103,00 & 83,80 & $\mathrm{AL}$ & 1,14 & 1,06 & 0,61 \\
\hline NVG & $\exp$ & 1,74 & 7,70 & 28,20 & 0,55 & 7,05 & 77,40 & $\mathrm{AL}$ & 4,35 & 0,65 & 0,26 \\
\hline M100 & $\exp$ & 0,92 & 5,38 & 124,80 & 0,92 & 0,37 & 64,40 & MO & 0,52 & 0,98 & 0,68 \\
\hline PG & esf & $1,26.10^{2}$ & $3,95.10^{2}$ & 92,70 & 0,60 & $2,81.10^{10}$ & 67,00 & MO & 74,06 & 0,96 & 0,64 \\
\hline $\mathrm{P}$ & esf & 2,00 & 325,50 & 10,80 & 0,00 & $2,68.10^{3}$ & 99,60 & $\mathrm{AL}$ & 1,70 & 0,96 & 0,36 \\
\hline $\mathrm{K}$ & gau & 49,40 & 98,90 & 91,79 & 0,81 & 204,00 & 50,10 & MO & 5,68 & 0,85 & 0,46 \\
\hline $\mathrm{Ca}$ & epp & 0,15 & 0,15 & - & - & - & - & - & - & - & - \\
\hline $\mathrm{Mg}$ & esf & $3,6.10^{-4}$ & $7,00 \cdot 10^{-3}$ & 16,40 & 0,10 & $2,94 \cdot 10^{-6}$ & 95,00 & $\mathrm{AL}$ & 0,05 & 0,94 & 0,37 \\
\hline SB & epp & 0,21 & 0,21 & - & - & - & - & - & - & - & - \\
\hline $\mathrm{Al}$ & exp & $1,6.10^{-3}$ & $5,60 \cdot 10^{-3}$ & 46,20 & 0,52 & $3,61.10^{-6}$ & 71,00 & MO & 0,02 & 0,89 & 0,41 \\
\hline $\mathrm{H}+\mathrm{Al}$ & gau & 0,38 & 0,90 & 21,13 & 0,41 & 0,10 & 57,00 & MO & 0,28 & 0,94 & 0,40 \\
\hline $\mathrm{t}$ & epp & 0,19 & 0,19 & - & - & - & - & - & - & - & - \\
\hline $\mathrm{T}$ & gau & 0,50 & 1,16 & 20,26 & 0,27 & 0,27 & 57,00 & MO & 1,79 & 0,76 & 0,31 \\
\hline $\mathrm{V} \%$ & epp & 27,62 & 27,62 & - & - & - & - & - & - & - & - \\
\hline $\mathrm{m} \%$ & gau & 5,26 & 14,62 & 18,35 & 0,30 & 23,30 & 64,00 & MO & 0,70 & 0,96 & 0,38 \\
\hline $\mathrm{pH}$ & gau & 0,02 & 0,12 & 9,52 & 0,00 & $4,09 \cdot 10^{-6}$ & 82,00 & $\mathrm{AL}$ & 1,22 & $-1,27$ & 0,24 \\
\hline \multirow[t]{2}{*}{ MO } & epp & 0,27 & 0,27 & - & - & - & - & - & - & - & - \\
\hline & \multicolumn{11}{|c|}{ Semivariograma cruzado } \\
\hline $\mathrm{PG}=\mathrm{f}(\mathrm{NT})$ & gau & $-1,00$ & $-568,90$ & 83,83 & 0,66 & $9,79.10^{3}$ & 99,80 & $\mathrm{AL}$ & 50,40 & 0,72 & 0,58 \\
\hline$P G=f(K)$ & gau & $1,81.10^{4}$ & $4,31.10^{4}$ & 106,52 & 0,91 & $7,97.10^{9}$ & 58,00 & MO & 56,14 & 0,68 & 0,55 \\
\hline$P G=f(H+A l)$ & gau & $-142,00$ & $-494,90$ & 98,23 & 0,90 & $12,62 \cdot 10^{3}$ & 71,30 & MO & 56,54 & 0,68 & 0,55 \\
\hline$P G=f(m \%)$ & gau & $-176,00$ & $-1,46.10^{2}$ & 140,12 & 0,83 & $3,60.10^{3}$ & 88,00 & $\mathrm{AL}$ & 56,32 & 0,68 & 0,55 \\
\hline
\end{tabular}

${ }^{1} \mathrm{NP}$ : número de planta; NT: nota de incidência de galhas nas raízes; ALT: altura de planta; INS: altura de inserção da primeira vagem; NVG: número de vagem por planta; M100: massa de cem grãos; PG: produtividade de grãos de feijão ( $\left.\mathrm{kg} \mathrm{ha}^{-1}\right)$; P: Fósforo do solo $\left(\mathrm{mg} \mathrm{dm}^{-3}\right)$; K: Potássio do solo $\left(\mathrm{mg} \mathrm{dm}^{-3}\right)$; Ca: Cálcio do solo $\left(\mathrm{cmol}_{\mathrm{c}} \mathrm{dm}^{-3}\right)$; Mg: Magnésio do solo $\left(\mathrm{cmol}_{\mathrm{c}} \mathrm{dm}^{-3}\right)$; SB: Soma de bases $\left(\mathrm{cmol}_{\mathrm{c}} \mathrm{dm}^{-3}\right)$; Al: Alumínio do solo $\left(\mathrm{cmol}_{\mathrm{c}} \mathrm{dm}{ }^{-3}\right)$; $\mathrm{H}+\mathrm{Al}$ : acidez do solo $\left(\mathrm{cmol}_{\mathrm{c}}\right.$ $\left.\mathrm{dm}^{-3}\right)$; t: CTC efetiva $\left(\mathrm{cmol}_{\mathrm{c}} \mathrm{dm}^{-3}\right)$; T: CTC potencial $\left(\mathrm{cmol}_{\mathrm{c}} \mathrm{dm}^{-3}\right)$; pH: Potencial hidrogeniônico em água; V\%: Saturação por bases; m\%: Saturação por alumínio; MO: Matéria orgânica do solo $\left(\mathrm{g} \mathrm{kg}^{-1}\right)$, respectivamente. ${ }^{2}$ esf: modelo esférico; gau: modelo gaussiano; exp: modelo exponencial; epp: efeito pepita pura; $\mathrm{C} 0, \mathrm{C} 0+\mathrm{C}$ e Ao são respectivamente: efeito pepita, patamar e alcance; SQR: soma dos quadrados dos resíduos. ${ }^{3} \mathrm{ADE}$ : avaliador de dependência espacial, sendo MO: moderada dependência espacial e AL: alta dependência espacial.

As variáveis $\mathrm{NT}, \mathrm{K}, \mathrm{H}+\mathrm{Al}, \mathrm{T}, \mathrm{m} \%$ e $\mathrm{pH}$ foram ajustadas ao modelo de semivariograma gaussiano, já as variáveis ALT,
INS, NVG, M100 e Al foram ajustadas pelo modelo exponencial, e a PG, P e Mg pelo modelo esférico (Tabela 2). 
Para verificação da correlação espacial da $\mathrm{PG}$ com os atributos do solo e NT, testou-se a formação de semivariogramas cruzados. Com isso, verificou-se correlação espacial inversamente proporcional da $\mathrm{PG}$ com a NT, H+Al e $\mathrm{m} \%$ (Tabela 2). Confirmando que plantas com maiores incidências de nematoides e em locais com maiores teores de $\mathrm{Al}$ apresentaram as menores produtividades. Houve correlação espacial positiva entre a PG e o $\mathrm{K}$, indicando que em locais com maiores teores de potássio foram encontradas as maiores produtividades. Logo, teores de $\mathrm{Al}$ de até $0,39 \mathrm{cmol}_{\mathrm{c}} \mathrm{dm}^{-3}$ foram limitantes a produtividade do feijoeiro, e os menores teores de $\mathrm{K}$ encontrados no solo possivelmente restringiram a PG (Tabela 1).

Por meio do mapa de produtividade pôde-se verificar que a cultivar BRS Pérola apresentou as maiores produtividades, superiores a $2000 \mathrm{~kg} \mathrm{ha}^{-1}$ (Figura 2). A cultivar BRS Ametista apresentou o menor desempenho em PG (1342 kg ha-1), diferindo estatisticamente das demais. No entanto, verifica-se que a região de menor produtividade apresentou os menores teores de potássio, maior incidência de nematoides de galha nas raízes e maior teores de acidez potencial e $\mathrm{m} \%$.
A cultivar BRS Pérola apresentou a maior M100 com valor médio de 28,55 g. As cultivares BRS Estilo e BRS Notável não apresentaram diferença significativa para esse atributo (Figura 2). Para INS e ALT a cultivar BRS Notável o pior desempenho entre as cultivares, com médias abaixo de $20 \mathrm{~cm}$ e $50 \mathrm{~cm}$, respectivamente (Figura 2). As demais cultivares não diferiram estatisticamente entre si frente a estes atributos.

A cultivar BRS Estilo apresentou o maior número de plantas (NP), quando comparado as demais cultivares (Figura 2), com uma média de 31.071 plantas $\mathrm{ha}^{-1}$, diferente desta cultivar, ao associarmos o NP (Figura 2) aos atributos do solo explícitos na figura 3 , observa-se que os possíveis agentes causadores do baixo desempenho em PG da cultivar BRS Ametista são menor NP, maior NT e menor teor de K.

As notas de incidência de nematoides foram maiores nas regiões com menor disponibilidade de $\mathrm{K}$, e maiores teores de acidez potencial $(\mathrm{H}+\mathrm{Al})$ e $\mathrm{m} \%$, onde estavam localizadas as cultivares de feijoeiro BRS Estilo e BRS Ametista (Figura 3), mostrando que a associação destes fatores pode influenciar diretamente na NT, ambas cultivares não apresentaram diferença estatística.
(A)

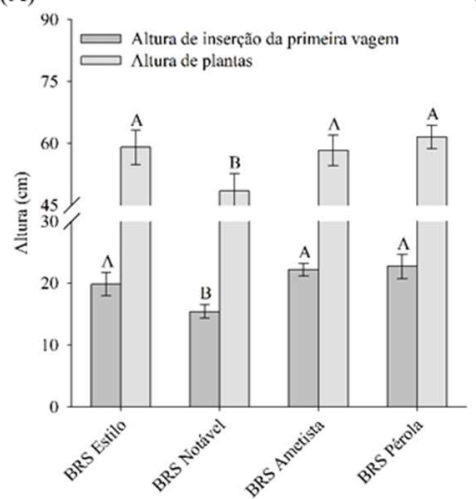

(D)
(B)

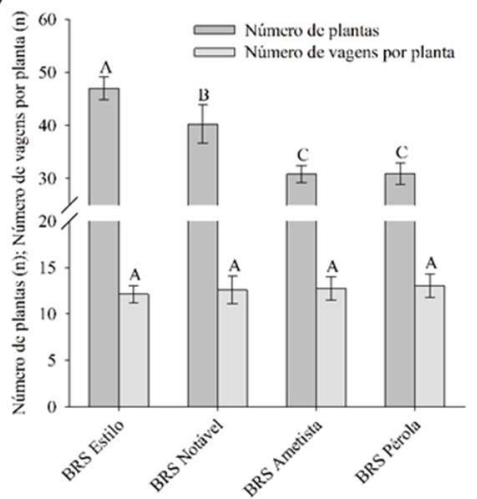

(C)

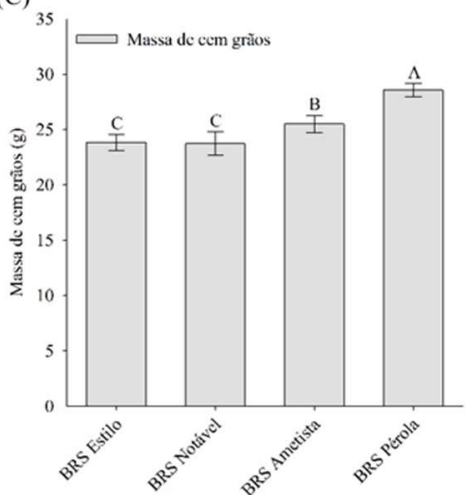

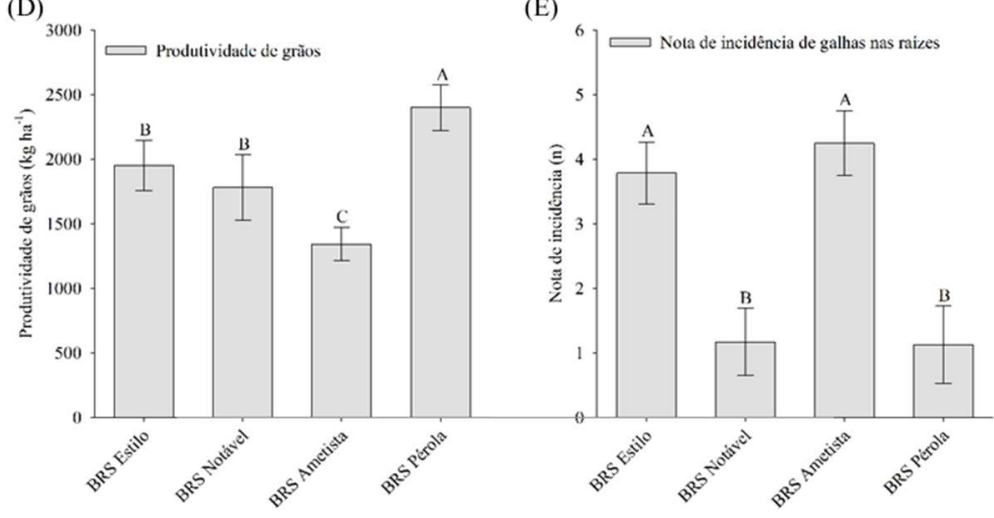

Figura 2. Comparação de médias dos atributos de planta de diferentes cultivares de feijão comum irrigado. Figure 2. Comparison of averages of plant attributes of different irrigated common bean cultivars.

\section{DISCUSSÃO}

A alta variabilidade dos atributos químicos do solo e dos atributos de planta puderam ser justificados pela amplitude dos valores de produtividade encontrados, devido os mesmos serem constituídos de quatro cultivares de feijoeiro, com produtividades entre 700 a $3044 \mathrm{~kg} \mathrm{ha}^{-1}$ (Tabela 1). A produtividade média obtida neste estudo foi de $1810 \mathrm{~kg} \mathrm{ha}^{-1}$, valor este superior à safra nacional de 2015 de $1290 \mathrm{~kg} \mathrm{ha}^{-1}$ (CONAB, 2016).

De acordo com a classificação proposta por Sousa; Lobato (2004), o valor médio de potássio se encontra inferior ao valor adequado, visto que em solos da região do Cerrado valores adequados estão entre $50,70 \mathrm{mg} \mathrm{dm}^{-3}$ e $78,00 \mathrm{mg} \mathrm{dm}^{-3}$, devido serem solos de caráter ácido e com regime pluviométrico elevado. De acordo com Oliveira et al. (2009) na cultura do feijão, o potássio é o nutriente extraído e exportado em maiores quantidades, por isso na maioria dos solos onde é explorado comercialmente são encontrados teores baixos desse nutriente.

Os valores de $\mathrm{Al}$ e $\mathrm{H}+\mathrm{Al}$ foram semelhantes aos encontrados por Souza et al. (2011), onde concluíram que o aumento da produtividade do feijão estava correlacionado com a redução dos teores de alumínio. Isso ocorre devido a $\mathrm{H}+\mathrm{Al}$ e 
o Al estarem ocupando as cargas do solo que poderiam estar sendo ocupadas por bases, criando uma condição de elevada acidez do solo, no qual segundo Silva et al. (2004), reduz o diâmetro radicular à custa do crescimento em comprimento, afetando assim, de forma indireta um ou mais atributos de planta, sendo M100, PG, ALT, e INS.

A respeito da NT, resultado semelhante foi encontrado por Baida et al. (2011), demonstrando que algumas cultivares apresentaram mais de 50\% do sistema radicular destruído pela formação de galhas oriundas da colonização de Meloidogyne sp. (MARTINS et al., 2009). A incidência de nematoides, também influenciou de forma negativa os atributos de planta PG, M100 e INS, corroborando com Inomoto et al. (2011), que devido à ação destes parasitas sob o impedimento na absorção de água e nutrientes pelas raízes das plantas, a partir da formação de galhas ou lesões necróticas, através do bloqueio dos feixes vasculares, limitando a absorção de água e nutrientes necessários ao desenvolvimento da cultura.

Tal limitação pode reduzir a translocação de nutrientes da parte radicular para a parte aérea, resultando assim na perda de biomassa, tendo como consequência direta a redução da massa de cem grãos e produtividade (ARAÚJO FILHO et al., 2010; WANDERLEY et al., 2007).

A maior incidência de nematoide nas raízes em áreas com baixo teor de potássio podem estar diretamente relacionados com sua suscetibilidade a doenças e parasitas. De modo que a limitação de nutriente, no caso potássio, possivelmente proporcionou maior suscetibilidade da planta a ser parasitada pelo nematoide Meloidogyne sp. KANDJI et al., 2001).
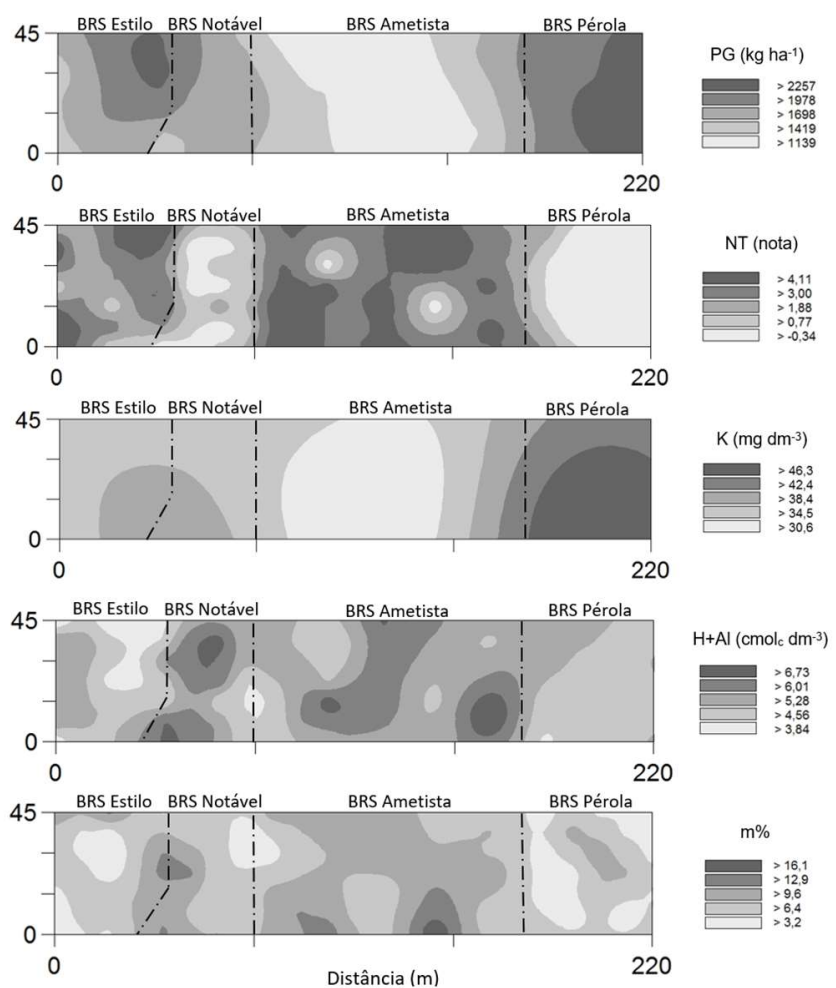

Figura 3. Mapas das propriedades químicas de um Latossolo Vermelho-Amarelo Distrófico e produtividade de grãos e nota de incidência de nematóides em cultivares de feijão irrigado.

Figure 3. Maps of the chemical properties of a Dystrophic Red Yellow Latossoland grain yield and incidence of nematodes in irrigated bean cultivars.

\section{CONCLUSÕES}

Nas condições em que o experimento foi realizado, a cultivar de feijão BRS Pérola apresentou a maior produtividade de grãos.

As cultivares BRS Pérola e BRS e BRS Notável apresentaram a menor incidência de nematoides.

Teores de $\mathrm{H}+\mathrm{Al}, \mathrm{m} \%$, alta incidência de nematoides e baixos teores de $\mathrm{K}$ foram negativamente correlacionados a variabilidade espacial da produtividade de grãos de feijão.

\section{AGRADECIMENTOS}

À EMBRAPA Agrossilvipastoril em especial à Dulândula Silva Miguel Wruck, Valéria de Oliveira Faleiro, Antônio Gonzaga Damasceno e Anizia Fátima Francisco Betti pela ajuda nas análises.

\section{REFERÊNCIAS}

AMADO, T. J. C.; PES, L. Z.; LEMAINSKI, C. L.; SCHENATO, R. B. Atributos químicos e físicos de Latossolos e sua relação com os rendimentos de milho e feijão irrigados. Revista Brasileira de Ciências do Solo, Viçosa, v. 33, n. 4, p. 831-843, 2009. DOI: http://dx.doi.org/10.1590/S0100-06832009000400008

ANA_AGÊNCIA NACIONAL DE ÁGUAS. Levantamento da Agricultura Irrigada por Pivôs Centrais no Brasil. 2014. Disponível em: $<$ http://www2.ana.gov.br/Paginas/imprensa/noticia.aspx? List $=$ ccb75a86-bd5a-4853-8c76 cc46b7dc89a1\&ID=13007>. Acesso em: $14 \mathrm{dez} 2016$.

ARAÚJO FILHO, J. V.; INOMOTO, M. M.; GODOY, R.; FERRAZ, L. C. C. B. Resistência de linhagens de feijãoguandu a Meloidogyne javanica. Nematologia Brasileira, Piracicaba, v. 34, n. 2, p. 75-81, 2010.

BAIDA, F. C.; SANTIAGO, D. C.; TAKAHASHI, L. S. A.; ATHANÁZIO, J. C.; CADIOLI, M. C.; LEVY, R. M. Reação de linhagens de feijão-vagem ao Meloidogyne javanica e M. paranaensis em casa-de-vegetação. Acta Scientiarum Agronomy, Maringá, v. 33, n. 2, p. 237-241, 2011.

DOI: https://dx.doi.org/10.4025/actasciagron.v33i2.6146

CHRISTIE, D. Resampling with Excel. Teaching Statistics, Sheffield, v. 26, n. 1, p. 9-14, 2004. DOI: http://dx.doi.org/10.1111/j.1467-9639.2004.00136.x

\section{CONAB_COMPANHIA NACIONAL DE} ABASTECIMENTO. Acompanhamento da safra brasileira. 2016. Disponível em: $<$ http://www.conab.gov.br/OlalaCMS/uploads/arquivos/1 6_06_09_09_00_00_boletim_graos_junho_2016_final.pdf $>$. Acesso em: 10 jul 2016.

CONAB_COMPANHIA NACIONAL DE ABASTECIMENTO. Acompanhamento da safra brasileira. 2017.2 Disponível em: <www.conab.gov.br/.../17_03_14_15_28_33_boletim_gr aos_marco_2017bx.pdf $>$. A Acesso em 18 mar 2018.

DALCHIAVON, F. C.; CARVALHO, M. P.; FREDDI, O. S.; ANDREOTI, M.; MONTANARI, R. Variabilidade espacial da produtividade do feijoeiro correlacionada com atributos químicos de um Latossolo Vermelho Distroférrico sob sistema de semeadura direta. Bragantia, Campinas, v. 70, n. 4, p. 908-916, 2011. DOI: http://dx.doi.org/10.1590/S0006-87052011000400025

EMBRAPA_EMPRESA BRASILEIRA DE PESQUISA AGROPECUÁRIA. Manual de métodos de análise de solo. 2011. Disponível em: 
$<$ http://ainfo.cnptia.embrapa.br/digital/bitstream/item/104 933/1/Manual-de-Mtodos-de-Anilise-de-Solo.pdf> Acesso em: 03 jan 2016.

EMBRAPA EMPRESA BRASILEIRA DE PESQUISA AGROPECUÁRIA. Brazilian Soil Classification System. 2018. Disponível em: https://www.embrapa.br/busca-de-publicacoes//publicacao/1094001/brazilian-soil-classification-system> Acesso em 25 set 2018.

GOMES, E. P.; JORDAN, R. A.; MOTOMIYA, A. V. da A.; PADUA, J. B.; BISCARO, G. A.; GEISENHOFF, L. O. Análise econômica e viabilidade energética da cultura do feijoeiro comum sob irrigação. Revista Brasileira de Engenharia Agrícola e Ambiental, Campina Grande, v.1 7, n. $8, \quad$ p. $835-842$, 2013. DOI: http://dx.doi.org/10.1590/S1415-43662013000800006

HOU, X. Q.; LI, R.; JIA, Z. K.; HAN, Q. F.; YANG, B. P.; NIE J. F. Effects of rotational tillage practices on soil structure, organic carbon concentration and crop yields in semi-arid areas of northwest China. Soil Use Management, v. 28, n. 4, p. 551-558,2012. DOI: https://dx.doi.org/10.1111/j.1475-2743.2012.00429.x

INOMOTO, M. M.; SIQUEIRA, K. M. S.; MACHADO, A. C. Z. Sucessão de Cultura sob Pivô Central para Controle de Fitonematóides: variação populacional, patogenicidade e estimativa de perdas. Tropical Plant Pathology, Brasília, v. 36, n. 3, p. 178-185, 2011 .

KANDJ, S. T.; OGOL, C. K. P. O.; ALBRECHT, A. Diversity of plant-parasitic nematodes and their relationships with some soil physico-chemical characteristics in improved fallows in western Kenya. Applied Soil Ecology, Amsterdam, v. 18, n. 2, p. 143-157, 2001. DOI: https://dx.doi.org/10.1016/S09291393(01)00157-3

MACHADO, A. C. Z. Nematoides em feijão: perdas de $\mathbf{1 0 \%}$ podem chegar a 50\%. Portal do agronegócio, 2011. Disponível em: $<$ http://nematologia.com.br/2011/12/nematoides-emfeijao-perdas-de-10-podem-chegar-a-50/>. Acesso em: 17 nov 2016.

MARTINS, M.; FONSECA, L.; MELO, L.; OLIVEIRA, D.; ALVIM, K.; SANTANA, D. Avaliação de genótipos de feijoeiro comum do grupo comercial carioca cultivados nas épocas das águas e do inverno em Uberlândia, Estado de Minas Gerais. Acta Scientiarum, Maringá, v.31, n.1, p. 23-28, 2009. DOI http://dx.doi.org/10.4025/actasciagron.v31i1.6606

MELLO, J. M.; COUTO, E. G.; AMORIM, R. S. S.; CHIG, L. A.; JOHNSON, M. S.; LOBO, F. A. Dinâmica dos atributos físico-químicos e variação sazonal dos estoques de carbono no solo em diferentes fitofisionomias do pantanal Norte Mato-grossense. Revista Árvore, Viçosa, v. $39, \quad$ n. 2 , p. $325-336,2015$. DOI: http://dx.doi.org/10.1590/0100-67622015000200012

MONTANARI, R.; CARVALHO, M. P.; ANDREOTTI, M.; DALCHIAVON, F. C.; LOVERA, M. A. O. H. Aspectos da produtividade do feijão correlacionados com atributos físicos do solo sob elevado nível tecnológico de manejo. Revista Brasileira de Ciência do Solo, Viçosa, v. 34, n. 6, p. 1811-1822, 2010. DOI: http://dx.doi.org/10.1590/S0100-06832010000600005

OLIVEIRA, A. P. de; SILVA, J. A. da; LOPES, E. B.; SILVA, E. É.; ARAUJO, L. E. A.; RIBEIRO, V. V. Rendimento produtivo e econômico do feijão-caupi em função de doses de potássio. Ciência e Agrotecnologia, Lavras, v. 33, n. 2, p. 629-634, 2009. DOI: http://dx.doi.org/10.1590/S141370542009000200042

PIMENTEL-GOMES, F.; GARCIA, C. H. Estatística aplicada a experimentos agronômicos e florestais. Piracicaba: FEALQ, 2002. 309 p.

SILVA, L. M.; LEMOS, L. B.; CRUSCIOL, C. A. C.; FELTRAN, J. C. S. Sistema radicular de cultivares de feijão em resposta à calagem. Pesquisa Agropecuária Brasileira, Brasília, v. 39, n. 7, p. 701-707, 2004. DOI: http://dx.doi.org/10.1590/S0100-204X2004000700012

SILVA, P. C. M.; CHAVES, L. H. G. Avaliação e variabilidade espacial de fósforo, potássio e matéria orgânica em Alissolos. Revista Brasileira de Engenharia Agrícola e Ambiental, Campina Grande, v. 5, n. 3, p. 431436, 2001. DOI: http://dx.doi.org/10.1590/S141543662001000300011

SOUSA, D. D.; LOBATO, E. Cerrado: correção do solo e adubação. Embrapa Cerrados: Planaltina, 2004. 416 p.

SOUZA G. S.; SOUZA, Z. M.; SILVA, R. B.; BARBOSA, R. S.; ARAÚJO, F. S. Effects of traffic control on the soil physical quality and the cultivation of sugarcane. Revista Brasileira de Ciência do Solo, Viçosa, v. 38, n. 1, p. 13546, 2014. DOI: http://dx.doi.org/10.1590/S010006832014000100013

SOUZA, H. A. de; HERNANDES, A.; ROMUALDO, L. M.; ROZANE, D. E.; NATALE, W.; BARBOSA, J. C. Folha diagnóstica para avaliação do estado nutricional do feijoeiro. Revista Brasileira de Engenharia Agrícola e Ambiental, Campina Grande, v. 15, n. 12, p. 1243-1250, 2011. DOI: http://dx.doi.org/10.1590/18071929/agriambi.v18n11p1102-1109

TAYLOR, A. L.; SASSER, J. N. Biology, identification and control of root-knot nematodes (Meloidogyne species). Raleigh: NCSU \& USAID, 1978. $111 \mathrm{p}$.

WANDERLEY, M. J. A.; WANDERLEY, P. A.; ATHAYDE FILHO, P. F. de; SANTOS, J. M. dos; PEREIRA, E. R. Resistência genética do feijão caupi ao nematoide Meloidogyne javanica. Revista Brasileira Agroecologia, Pelotas, v. 2, n. 1, p. 1377-1380, 2007. 